JOURNAL OF

SYNCHROTRON

RADIATION

ISSN 1600-5775

Received 19 July 2016

Accepted 2 November 2016

Edited by M. Yabashi, RIKEN SPring-8 Center, Japan

Keywords: X-ray grating interferometry; wavefront metrology; X-ray optics; XFELs.

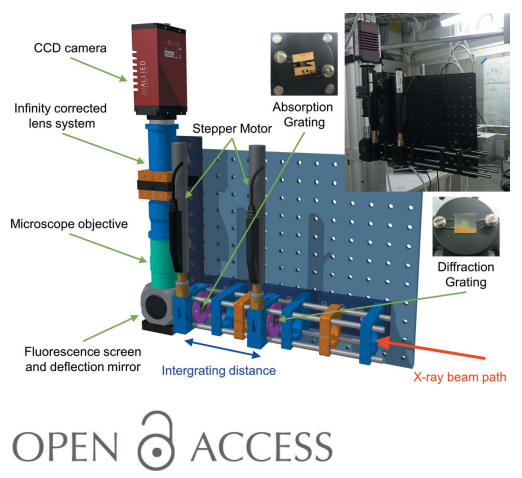

\section{$X$-ray grating interferometer for in situ and at-wavelength wavefront metrology}

Yves Kayser,* Christian David, Uwe Flechsig, Juraj Krempasky, Volker Schlott and Rafael Abela

Paul Scherrer Institut, 5232 Villigen PSI, Switzerland. *Correspondence e-mail: yves.kayser@psi.ch

A wavefront metrology setup based on the X-ray grating interferometry technique for spatially resolved, quantitative, in situ and at-wavelength measurements of the wavefront at synchrotron radiation and hard X-ray freeelectron laser beamlines is reported. Indeed, the ever-increasing demands on the optical components to preserve the wavefront shape and the coherence of the delivered X-ray beam call for more and more sensitive diagnostic instruments. Thanks to its angular sensitivity, X-ray grating interferometry has been established in recent years as an adequate wavefront-sensing technique for quantitatively assessing the quality of the X-ray wavefront under working conditions and hence for the in situ investigation of X-ray optical elements. In order to characterize the optical elements at any given beamline by measuring the aberrations introduced in the wavefront, a transportable X-ray grating interferometry setup was realised at the Swiss Light Source (SLS). The instrument, which is expected to be a valuable tool for investigating the quality of the X-ray beam delivered at an endstation, will be described hereafter in terms of the hardware setup and the related data analysis procedure. Several exemplary experiments performed at the X05DA Optics beamline of the SLS will be presented.

\section{Introduction}

Following the introduction of X-ray grating interferometry (XGI) (David et al., 2002; Momose et al., 2003), its potential for X-ray wavefront metrology purposes was quickly recognized. Indeed, the method's degree of angular accuracy is experimentally demonstrated to be of the order of tens of nanoradians (Weitkamp et al., 2005a) while the requirements regarding the longitudinal and transverse coherence of the beam as well as the photon flux are quite moderate (Weitkamp et al., 2005b; Momose et al., 2006). This makes XGI suitable for investigating the $\mathrm{X}$-ray wavefront at synchrotron radiation or $\mathrm{X}$-ray free-electron laser (XFEL) facilities in order to detect $\mathrm{X}$-ray wavefront distortions introduced by the optical elements. The need for sensitive wavefront metrology tools is driven by the increased quality of the X-ray radiation delivered by the operational XFELs (Emma et al., 2010; Ishikawa et $a l ., 2012)$ and the upcoming diffraction-limited storage rings (Eriksson et al., 2014; Yabashi et al., 2014). To profit from the brightness and coherence of the delivered X-ray beam in spectroscopy, diffraction, scattering or imaging experiments, the optical elements have to be able to preserve the wavefront (or phase) properties. The challenge to steer diffractionlimited and coherence-preserved X-ray beams from the source towards the experiment defines extraordinary high-quality requirements on the production of the X-ray optics. In particular, the figure errors of the mirrors have to be below 
$100 \mathrm{nrad}$ root-mean-square which is close to the current limit of state-of-the-art visible-light metrology with interferometers or profilometers (Goldberg et al., 2013; Idir et al., 2013; Siewert et al., 2014; Yashchuk et al., 2015) and close to the limits of the currently available optics manufacturing technology (Thiess et al., 2010; Yamada et al., 2015; Störmer et al., 2016).

Among the available at-wavelength wavefront diagnostic methods (Sawhney et al., 2013) like the pencil beam technique (Hignette et al., 1997; Sutter et al., 2012), the Hartmann sensor (Idir et al., 2014) or the speckle tracking approach (Berujon et al., 2012; Wang et al., 2015), XGI is nowadays a well established quantitative diagnostic method. It is used for assessing the quality of optical elements and detecting imperfections emanating from the X-ray beam itself or from X-ray optical elements. For metrology purposes XGI was applied to the investigation of multilayer mirrors (Weitkamp et al., 2005a; Rack et al., 2010), monochromators (Diaz et al., 2010; Rutishauser et al., 2013a), Fresnel zone plates (Wang et al., $2013 a, b$ ) and a single compound refractive lens (Rutishauser $e t$ al., 2011). It was also suggested to use XGI for the in situ monitoring of mirror surface finishing (Ziegler et al., 2007). Thus, XGI proves to be complementary to ex situ visible-light diagnostic tools like the long-trace profilometer (Yashchuk et al., 2010; Flechsig et al., 2013), the nanometer optical component measuring machine (Alcock et al., 2010; Siewert et al., 2011) or the Fizeau interferometer (Hariharan, 1997). These are necessary and essential metrology instruments for the investigation and characterization of X-ray optical elements, as well as for cross-check, deterministic correction or validation measurements of optical surfaces during the manufacturing process or after delivery. Furthermore, calibration measurements and pre-alignment or mechanical adjustments prior to the installation of an optical element at a beamline can be conveniently realised using ex situ methods. Possible drawbacks of these methods are difficulties with steeply curved aspherical surfaces, the requirement of a reference surface for calibration or the limitation to one-dimensional measurements. Furthermore, the influence of the beamline environment, which often limits the performance of the optical elements, cannot be assessed by means of ex situ metrology measurements.

Because XGI is an at-wavelength and in situ method, it permits to characterize optical elements under operational conditions in one or two dimensions and to detect in a non-invasive manner wavefront degradation caused by radiation damage, heat load or mechanical mounting (mounting, clamping, stress, vibrations). Indeed, XGI was used to characterize the heat bump of a monochromator subject to different incident radiation powers (Rutishauser et al., 2013a) and as a feedback tool to correct under working

Figure 1 conditions residual wavefront errors by means of adaptive optics in order to optimize the optic's performance (Wang et al., 2014; Marathe et al., 2014a). The coherence properties of the delivered X-ray beam have been studied as well by means of XGI (Pfeiffer et al., 2005; Diaz et al., 2010; Marathe et al., 2014b; Shi et al., 2014; Assoufid et al., 2016). With respect to XFEL metrology applications, the single-shot capability of XGI is extremely interesting. Mirrors and monochromators as well as the longitudinal position jitter of the photon source at the Linac Coherent Light Source (Emma et al., 2010) and at the SPring-8 Angstrom Compact Free-Electron Laser (Ishikawa et al., 2012) were investigated by means of XGI (Rutishauser et al., 2012; Kayser et al., 2014, 2016).

In the following we describe a transportable X-ray grating interferometer and show typical exemplary applications in order to demonstrate the potential of XGI and to investigate its limitations. A comparable realisation of a portable XGI setup realised at the Advanced Photon Source was published very recently (Assoufid et al., 2016). The setup described hereafter was realised for measurements in the moiré mode in order to profit from the single-shot capabilities in view of measurements at XFELs. For the sake of completeness and consistency the principle of XGI and the required analysis steps of the experimental data will also be briefly discussed.

\section{Principle of XGI}

XGI is based on the detection of a periodic interference pattern created by the interference of the X-ray beams diffracted by a grating into positive and negative orders (Fig. 1). Downstream of the diffraction grating the interference between the diffracted X-ray beams produces a Talbot carpet characterized by planes of high-intensity contrast at discrete and periodic distances (Suleski, 1997). The grating, in its one-dimensional version, is a line structure and, in its twodimensional version, is a mesh pattern. The height of the

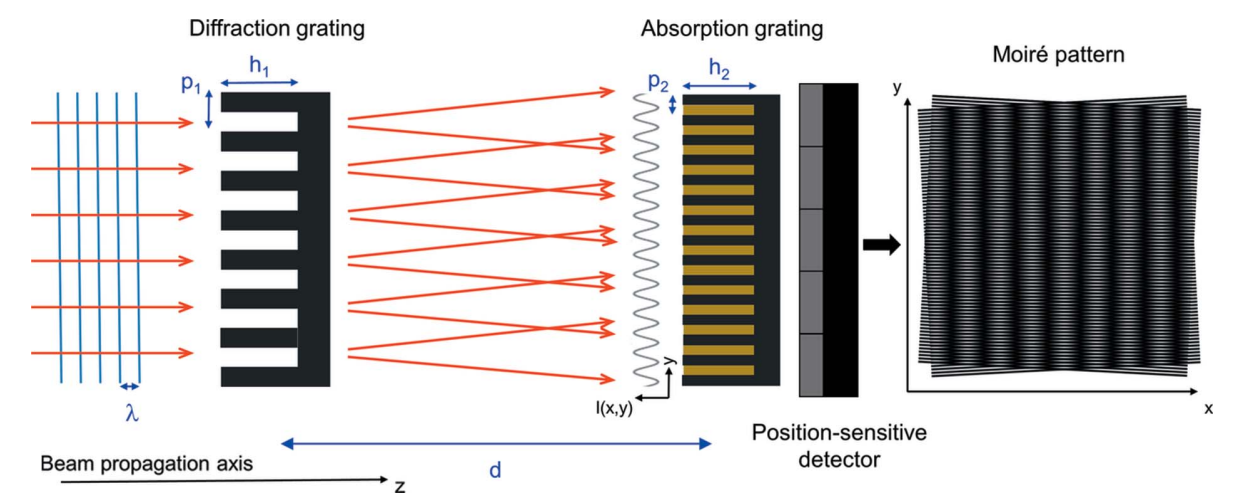

Schematic illustration of the principle of XGI in the moiré mode. The diffraction of an incident plane wave into positive and negative diffraction orders (propagating in the first order at an angle of $\lambda / p_{1}$ with respect to the original propagation direction) by the diffraction grating gives rise to an interference pattern. The diffraction grating induces a phase shift of $h_{1}(2 \pi \delta) / \lambda$ where $\delta$ is the wavelength-dependent decrement in the real part of the refractive index of the grating material. The absorption grating acts as a transmission mask. For small rotation angles of one grating with respect to the other ( $3^{\circ}$ with respect to the horizontal axis in the figure), the resulting moiré pattern can conveniently be imaged using an adequate camera system, whereas the spatial modulations with a high frequency cannot be resolved. 
grating structures is typically chosen such that it causes a $\pi / 2$ or a $\pi$ phase shift.

The best diffraction efficiency of the diffracting grating into the \pm 1 st diffraction order is obtained with binary $\pi$ phase shifting gratings and a duty cycle of 0.5 (Weitkamp et al., 2012). For such a diffraction grating and considering plane waves, the planes with the best contrast for the constructive interference pattern are at the so-called fractional Talbot orders $d_{\mathrm{n}}=$ $n p_{1}^{2} /(8 \lambda)$ ( $n$ being odd-numbered, $\lambda$ the wavelength and $p_{1}$ the periodicity of the diffraction grating) and the periodicity of the interference pattern is equal to $p_{1} / 2$. Most position-sensitive detectors, however, do not present a sufficient resolution to spatially resolve the interference pattern. To this prospect a second grating with absorbing structures having a pattern and a periodicity matching that of the interference pattern is positioned immediately in front of the detector. Its role is to serve as a transmission mask and it can be used in two different ways. In a first configuration the absorbing structures are aligned parallel to the interference pattern in terms of angular orientation around the beam propagation axis. A positional scan in the direction transverse to the grating structures over the course of one period of the absorption grating provides a measurement of the position-dependent intensity oscillations within each pixel. The pixel size defines then the spatial resolution with which the interference pattern is measured. This approach is called the phase-stepping mode in XGI.

The alternative method is based on rotating the diffracting and absorbing gratings with respect to each other around the optical axis. The moiré pattern resulting from the superposition of the structures from the absorption grating with the interference pattern generated by the diffraction grating has a periodicity coarse enough to be resolved by the positionsensitive detector. This approach is called the moiré mode in XGI. Mathematically the moiré pattern $I_{\mathrm{m}}(x, y)$ is described by

$$
I_{\mathrm{m}}(x, y)=\cos \left[\frac{2 \pi}{p_{\mathrm{m} x}} x+\frac{2 \pi}{p_{\mathrm{m} y}} y\right],
$$

with

$$
p_{\mathrm{m} x}=\frac{p_{2}}{\sin \beta_{2}-\left(M_{0} / M\right) \sin \beta_{1}}
$$

and

$$
p_{\mathrm{m} y}=\frac{p_{2}}{\cos \beta_{2}-\left(M_{0} / M\right) \cos \beta_{1}},
$$

where $p_{2}$ represents the periodicity of the absorption grating pattern, $M$ and $M_{0}$ stand for the experimental and the design magnification of the XGI setup, $\beta_{1}$ and $\beta_{2}$ are the tilt angles of the diffraction and absorption grating structures in the detection plane reference system and $p_{\mathrm{m} x}$ and $p_{\mathrm{m} y}$ correspond to the moiré fringe periodicity in the horizontal and vertical directions, respectively. When tuning the periodicity of the moiré pattern a trade-off between spatial resolution (improves with shorter periodicity) and angular sensitivity (increases with larger periodicity) of the measurement has to be made.
Compared with the phase-stepping approach, the moiré mode is less sensitive towards magnification issues resulting in residual moiré fringes. When considering divergent X-ray beams instead of plane waves, the interference pattern periodicity (and also the fractional Talbot order distances) have in principle to be corrected for by geometrical magnification factors (Weitkamp et al., 2004). The main advantage of the moiré mode, however, is the possibility for single-shot measurements resulting in shorter total acquisition times compared with the phase stepping approach. With respect to possible applications at XFELs, the moiré mode was therefore the configuration of choice for the realised X-ray grating interferometer, despite its lower spatial resolution.

In X-ray wavefront metrology applications, the X-ray wavefront incident on the grating interferometer can be reconstructed from a single image of the moiré pattern and aberrations introduced into the wavefront by optical elements can be detected. In the case of a plane wave incident on the X-ray grating interferometer a set of parallel fringes would ideally be measured (for one-dimensional gratings). Any distortion in the recorded periodic interference pattern, e.g. a local lateral shift or a curvature in the pattern, reveals a locally modified propagation direction of the incident X-rays caused by the optical element under investigation. In this respect it is important that the grating structures are free of any distortions since the quality of the gratings directly impacts the measurement in terms of the quality of the recorded data and the following interpretation. This cannot be corrected for in the reconstruction of the wavefront since it usually is not possible to separate interference pattern distortions induced by wavefront aberrations from those due to grating structure defects with a flat-field measurement. Well fabricated gratings of good quality which do not present any deviations from their design are thus the key component of any XGI metrology setup.

\section{XGI setup}

The gratings are usually fabricated from thinned $\mathrm{Si}$ wafers because of their flatness, smoothness and stiffness, and because of the relatively low absorption coefficient of Si. For the absorption grating, the grating trenches are usually filled with gold because of the large absorption cross-sections in the $\mathrm{keV}$ regime. Both $\mathrm{Si}$ and $\mathrm{Au}$ are commonly used in microfabrication processes and their properties during processing are well known. The challenge in the grating production is, especially for the absorption grating, the required high aspect ratio for hard X-ray energies and the small periodicity for better sensitivity. Moreover, the grating structures need to be positioned and aligned with high accuracy over the area covered by the grating pattern. A placement accuracy of the order of $1 \%$ of the line width can be achieved by current electron beam writer systems. If the latter supports a continuous path writing mode this allows furthermore to avoid stitching errors in the electron-beam lithography process (Schnabel \& Kley, 2001) while the widths of the lines which 
are written are controlled by the dose and the defocusing of the electron beam (David \& Hambach, 1999). The grating structures are subsequently transferred into the $\mathrm{Si}$ wafer by means of an etching process. For one-dimensional grating patterns this can be anisotropic wet etching (David et al., 2007) while for two-dimensional patterns deep reactive ion etching has to be used (Rutishauser et al., 2013b). While this marks the last step in the production of the diffraction grating, the fabrication of the absorption grating needs a further processing step (David et al., 2007; Rutishauser et al., 2013b). Either the grating trenches are filled with $\mathrm{Au}$ by electroplating (Rutishauser et al., 2013b) or the grating pattern is conformally covered by Au to produce a frequency doubled grating of gold structures (David et al., 2007). In both cases the grating trenches for the absorption grating have to be deep enough to deposit enough $\mathrm{Au}$ for a sufficiently large absorption of the incident X-ray photons. This improves the fringe contrast and the visibility. A detailed description of the grating production process is given by David et al. (2007) and Rutishauser et al. (2013b). Alternatively, gratings can be produced by the LIGA technique (Reznikova et al., 2008; Noda et al., 2008) or metal assisted chemical etching (Romano et al., 2016), respectively, the absorption grating can be replaced by a structured scintillator (Rutishauser et al., 2011).

The line gratings used for the exemplary measurements with one-dimensional sensitivity at the X05DA Optics beamline (Flechsig et al., 2009) of the Swiss Light Source (SLS) were designed for photon energies of $8.3 \mathrm{keV}$ and $12.4 \mathrm{keV}$. The gratings were manufactured from $250 \mu \mathrm{m}$-thick polished Si $\langle 100\rangle$ substrates which were thinned by deep reactive ion etching. The diffraction grating had a duty cycle of 0.5 and a periodicity of $4 \mu \mathrm{m}$ and covered an area of $4 \mathrm{~mm} \times 4 \mathrm{~mm}$. The absorption grating had the same area and duty cycle as the diffraction grating and a periodicity of $2 \mu \mathrm{m}$. The height of the gold structures was $\sim 10 \mu \mathrm{m}$. The grating parameters and their homogeneity throughout the grating pattern area were confirmed by scanning electron microscopy. The gratings were fixed by clamps made from tungsten wires on a flat mounting surface with a central bore for the passage of the X-ray beam (Fig. 2). The mounting surfaces were themselves fixed to a rotary support for the orientation of the grating surface around the beam propagation axis within an angular range of $20^{\circ}$. The rotary supports were attached via $300 \mathrm{~mm}$-long hardened stainless steel rods to holders. The mounting system using rods allows for a flexible adjustment, only limited by the position of the holders, of the distance between the diffraction and absorption gratings while ensuring the parallelism of the grating surface planes. Moreover, independent vibrations of the gratings are minimized. The rotary supports were motorized with actuators having a minimum incremental motion of $0.1 \mu \mathrm{m}$ and an on-axis accuracy of $\pm 5 \mu \mathrm{m}$. This converts to a minimum angular incremental motion of $0.00024^{\circ}$ and an angular accuracy of $\pm 0.012^{\circ}$. The actuators were connected to a universal multi-axis motion controller and connected through an Ethernet interface to the control PC. The motion control system is compatible with the Experimental Physics and Industrial Control System (EPICS).

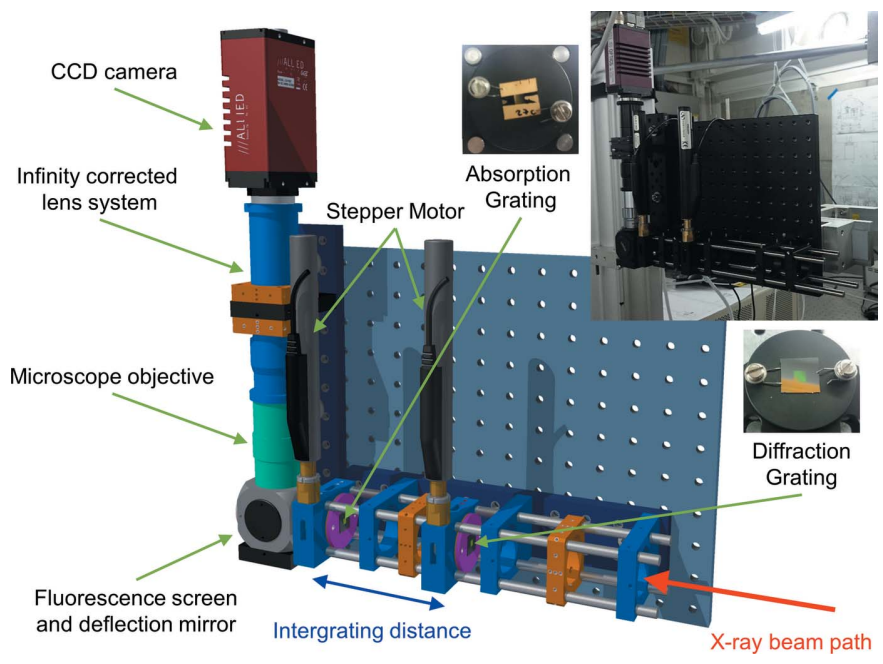

Figure 2

Schematic of the realised modular, transportable and self-contained XGI setup. The insets show the mounting of the gratings and the installed setup at the X05DA Optics beamline.

The moiré patterns were recorded using a modular twodimensional camera system coupled to a Ce-doped YAG screen $(10 \mathrm{~mm}$ in diameter, $30 \mu \mathrm{m}$ thickness $)$ and a deflection mirror (Fig. 2). The mirror avoids exposure of the camera's CCD chip to hard X-rays. The CCD camera allows for 14-bit acquisition and is directly connected through two bonded GigE interfaces to the control PC. This allows for a frame rate at full resolution of up to 112 images s $^{-1}$ which is compatible with the repetition rates of current XFELs in view of singleshot measurements. The chip consists of $1024 \times 1024$ pixels with a size of $5.5 \mu \mathrm{m}$. areaDetector software (Rivers, 2010), which is also based on EPICS, was used to control the camera. The camera was connected to an infinity-corrected lens system and a long-working-distance objective with twofold magnification. The effective pixel size of the optical system was found to be $2.857 \mu \mathrm{m}$ by means of an edge scan. The distance between the microscope objective and the fluorescence screen was adjusted to the focal distance of the objective with the help of a Siemens star. A resolution of about $3 \mu \mathrm{m}$ was assessed. Black kapton was mounted in front of the fluorescence screen in order to ensure a light-tight assembly. Dark images were acquired before each measurement to correct the recorded images for detector noise. The whole camera system was mounted on the same mounting plate as the interferometer. This ensures that the whole XGI setup constitutes a single, rigid and stiff mechanical unit with a high resistance against mechanical instabilities. The setup has moderate space requirements and can moreover be conveniently transported, mounted and aligned at different instances.

Both the motion control and the image acquisition system are independent of any beamline infrastructure and controlled through the Ethernet interfaces with one PC using the areaDetector and EPICS software to run measurements. Ultimately the realised XGI setup is flexible in use, transportable and self-contained. The only requirement on the beamline is that less than $500 \mathrm{~mm}$ of space along the beam propagation 
axis is available for installation of the XGI setup. In view of possible measurements in the phase stepping mode, as a complement to the moiré, one of the stepping motors can be replaced by a two-dimensional piezo stage which is supported by the motion control system.

The grating interferometry setup was assembled for the realisation of exemplary measurements at the X05DA Optics beamline of the SLS (Flechsig et al., 2009). A cryogenically cooled $\mathrm{Si}(111)$ channel-cut monochromator was used to monochromatize the X-ray beam. A bendable toroidal mirror allowed the X-ray beam to be focused to a smallest possible spot size of about $70 \mu \mathrm{m} \times 140 \mu \mathrm{m}$ in our case. The monochromator and the focusing mirror can be independently inserted or retracted from the X-ray beam. Since the vertical propagation angle of the $\mathrm{X}$-ray beam changes at the Optics beamline when inserting the mirror, the setup was mounted on the $2 \theta$ arm of a diffractometer which allowed, in combination with the vertically movable optical table, for a proper alignment with respect to the incident X-ray beam.

\section{Moiré pattern analysis}

For a quantitative analysis, the XGI setup first has to be calibrated by recording the moiré patterns at varying angular orientations between the diffraction and absorption gratings (Wang et al., 2011; Kayser et al., 2014). To do so, one of the gratings was scanned on an angular range centered on the position where the structures of both gratings are parallel to each other. For each measured moiré pattern the fringe frequency in the horizontal and vertical dimension was extracted by means of a two-dimensional fast Fourier transform (FFT) and a sub-pixel fitting routine using a twodimensional Gaussian function (Fig. 3). From the variation of the vertical component $p_{\mathrm{m} y}=1 / f_{\mathrm{m} y}$ with respect to the horizontal component $p_{\mathrm{m} x}=1 / f_{\mathrm{m} x}$ of the fringe periodicity (see Fig. 3), information on the XGI setup can be extracted through a minimization procedure (Wang et al., 2011; Kayser et al., 2014). Solving equations (2) and (3) for $\cos \beta_{1}$ and $\sin \beta_{1}$, $\cos \beta_{2}$ and $\sin \beta_{2}$, respectively, depending on whether the diffraction or the absorption grating was moved during the calibration scan, a parametric relationship can be obtained through $\cos ^{2} \gamma+\sin ^{2} \gamma=1$, where $\gamma$ represents the scanning parameter (either $\beta_{1}$ or $\beta_{2}$ ):

$$
\begin{aligned}
& \left(\frac{p_{2}}{p_{\mathrm{m} x}}-\sin \beta_{2}\right)^{2}+\left(\frac{p_{2}}{p_{\mathrm{m} y}}-\cos \beta_{2}\right)^{2}=\left(\frac{M_{0}}{M}\right)^{2}, \\
& \left(\frac{p_{2}}{p_{\mathrm{m} x}}+\frac{M_{0}}{M} \sin \beta_{1}\right)^{2}+\left(\frac{p_{2}}{p_{\mathrm{m} y}}+\frac{M_{0}}{M} \cos \beta_{1}\right)^{2}=1 .
\end{aligned}
$$

In the ensuing minimization procedure with respect to the experimental values $p_{\mathrm{m} x}^{i}$ and $p_{\mathrm{m} y}^{i}$ of the moire fringe period in the horizontal and vertical directions, the unknown parameters $M$ and $\beta_{2}$ [equation (4a)] and $\beta_{1}$ [equation (4b)], respectively, can be extracted,

$$
\begin{aligned}
& \text { Equation }(4 a) \rightarrow \min _{M, \beta_{2}} \sum_{i} \mid\left(\frac{p_{2}}{p_{\mathrm{m} x}^{i}}-\sin \beta_{2}\right)^{2} \\
& +\left(\frac{p_{2}}{p_{\mathrm{m} y}^{i}}-\cos \beta_{2}\right)^{2}-\left.\left(\frac{M_{0}}{M}\right)^{2}\right|^{k}, \\
& \text { Equation }(4 b) \rightarrow \min _{M, \beta_{1}} \sum_{i} \mid\left(\frac{p_{2}}{p_{\mathrm{m} x}^{i}}+\frac{M_{0}}{M} \sin \beta_{1}\right)^{2} \\
& \\
& +\left(\frac{p_{2}}{p_{\mathrm{m} y}^{i}}+\frac{M_{0}}{M} \cos \beta_{1}\right)^{2}-\left.1\right|^{k}
\end{aligned}
$$

Usually an $L_{1}$-norm minimization $(k=1)$ is realised because of its lesser sensitivity to experimental noise. Once the angular position of the grating which was kept fixed during the calibration scan is known, the angular orientation of the scanned grating can be obtained by means of equations (2) or (3) (Fig. 3). Furthermore, from the magnification $M$, the radius of curvature (ROC) can be calculated through $R=d /(M-1)$,

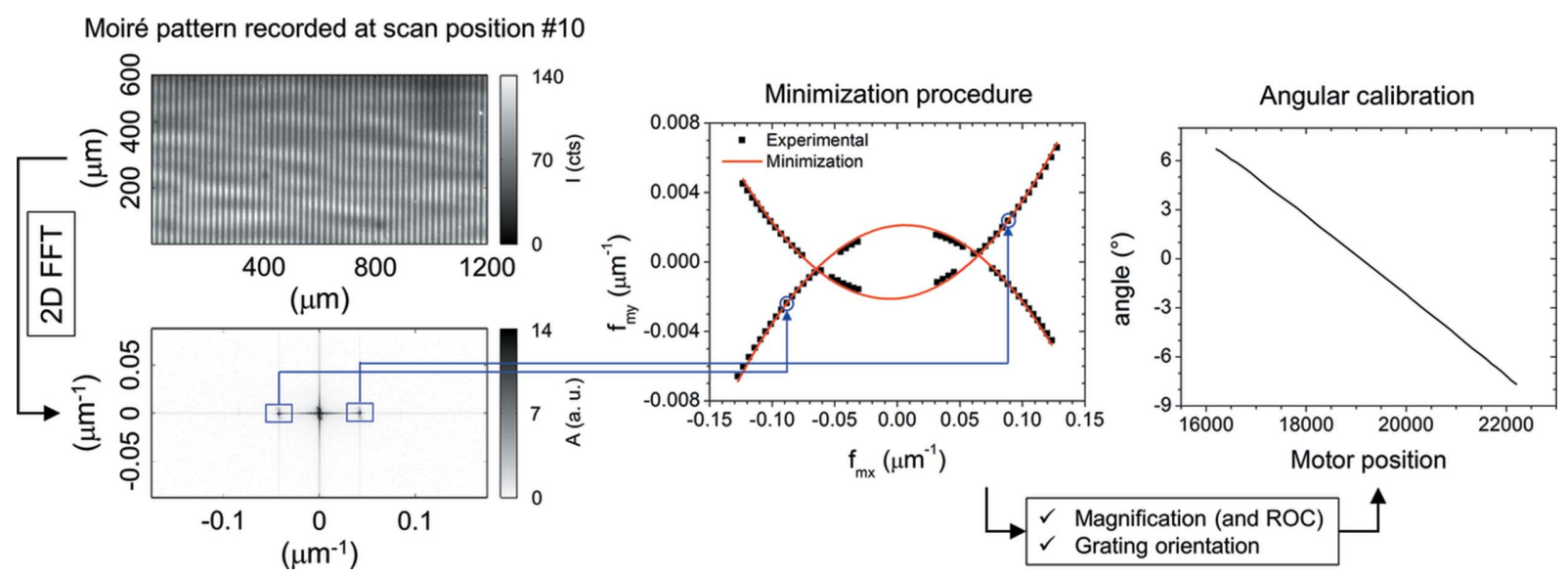

Figure 3

In the calibration procedure, the fringe frequencies of each moiré pattern are extracted via a two-dimensional FFT. The overall variation of the fringe frequency with angular orientation of the gratings with respect to each other allows us to calibrate the grating positions via a minimization procedure. More details can be found in the text. 
where $d$ stands for the distance between the diffraction and absorption grating.

In practice we scanned an angular range of $\pm 7.33^{\circ}$ with an angular stepping of $0.24^{\circ}$. The limit for the width of the angular range to be scanned is the increasingly small periodicity of the moiré fringe pattern when misaligning the grating structures with respect to each other: the moiré pattern cannot be resolved and the fringe frequencies not extracted. The angular stepping, and thus the number of data points in the minimization procedure, allows the standard error on the minimization parameters to be minimized. The selected angular stepping presented the best compromise between the returned standard error and the overall acquisition time for a calibration scan. Prior to the minimization procedure the data points for which the coordinates of the -1 st and +1 st Fourier order were not symmetric to each other were rejected. This was essentially necessary around the central position of the scan where the \pm 1 st orders are difficult to separate from the zeroth order.

Once the XGI setup is calibrated, the moiré patterns recorded at a single position of the diffraction and absorption gratings allow the incident X-ray wavefront to be investigated individually for each image. The processing of a measured moiré fringe pattern is based on Fourier analysis (Takeda et al., 1982). In the first step, as illustrated in Fig. 4, the moiré pattern is multiplied by a Hann window to reduce boundary effects which may occur in the consecutive FFT calculation because of the implicitly assumed periodic boundary conditions. Next, the first-order component is separated from the remaining frequencies by means of a band-pass filter (a box window since the zeroth and first-order components are well separated from each other) and shifted to the origin. The shift removes the carrier frequency contribution to the measured intensity pattern. In addition, it simplifies the following unwrapping procedure (Herráez et al., 2002) of the spatially resolved fringe phase $\Psi(x, y)$ obtained from the argument of the inverse FFT of the isolated first-order component. When line gratings are used for the measurement, the fringe phase is obtained in the direction parallel to the moire fringes. For mesh-type grating patterns, the analysis procedure is analogous, except that two-dimensional versions of the Hann window, the FFT and the band-pass filter are used. In the Fourier space, instead of one pair of first-order components, two pairs are present and the fringe phase is retrieved simultaneously in the horizontal and vertical dimensions from the respective first-order components.

Using the information of the calibration scan, the wavefront propagation angle in the horizontal or vertical direction can be calculated from the corresponding fringe phase (Rutishauser et al., 2012; Weitkamp et al., 2004),

$$
\begin{aligned}
& \alpha_{x}=\left(\frac{M_{0} \sin \beta_{\mathrm{f}}}{\sin \beta_{\mathrm{r}}}-1\right) \frac{x}{d}+\frac{p_{2} \sin \beta_{\mathrm{f}}}{2 \pi d \sin ^{2} \beta_{\mathrm{r}}} \Psi_{x}(x, y), \\
& \alpha_{y}=\left(\frac{M_{0} \cos \beta_{\mathrm{f}}}{\cos \beta_{\mathrm{r}}}-1\right) \frac{y}{d}+\frac{p_{2} \cos \beta_{\mathrm{f}}}{2 \pi d \cos ^{2} \beta_{\mathrm{r}}} \Psi_{y}(x, y),
\end{aligned}
$$

since $M_{0}$ and $d$ are known from the setup. Here, $\beta_{\mathrm{r}}$ represents the angle of the grating which was moved during the calibration scan and $\beta_{\mathrm{f}}$ the angle of the grating which was kept fixed. The aspherical component of the wavefront propagation angle, which may reveal aberrations in the wavefront from the expected spherical shape, can be retrieved by subtracting a first-order polynomial. The ROC in the horizontal or vertical direction of the incident wavefront can be calculated from the fringe phase (Rutishauser et al., 2012; Weitkamp et al., 2004) or from the slope of the wavefront propagation angle (Kayser $e t$ al., 2014).
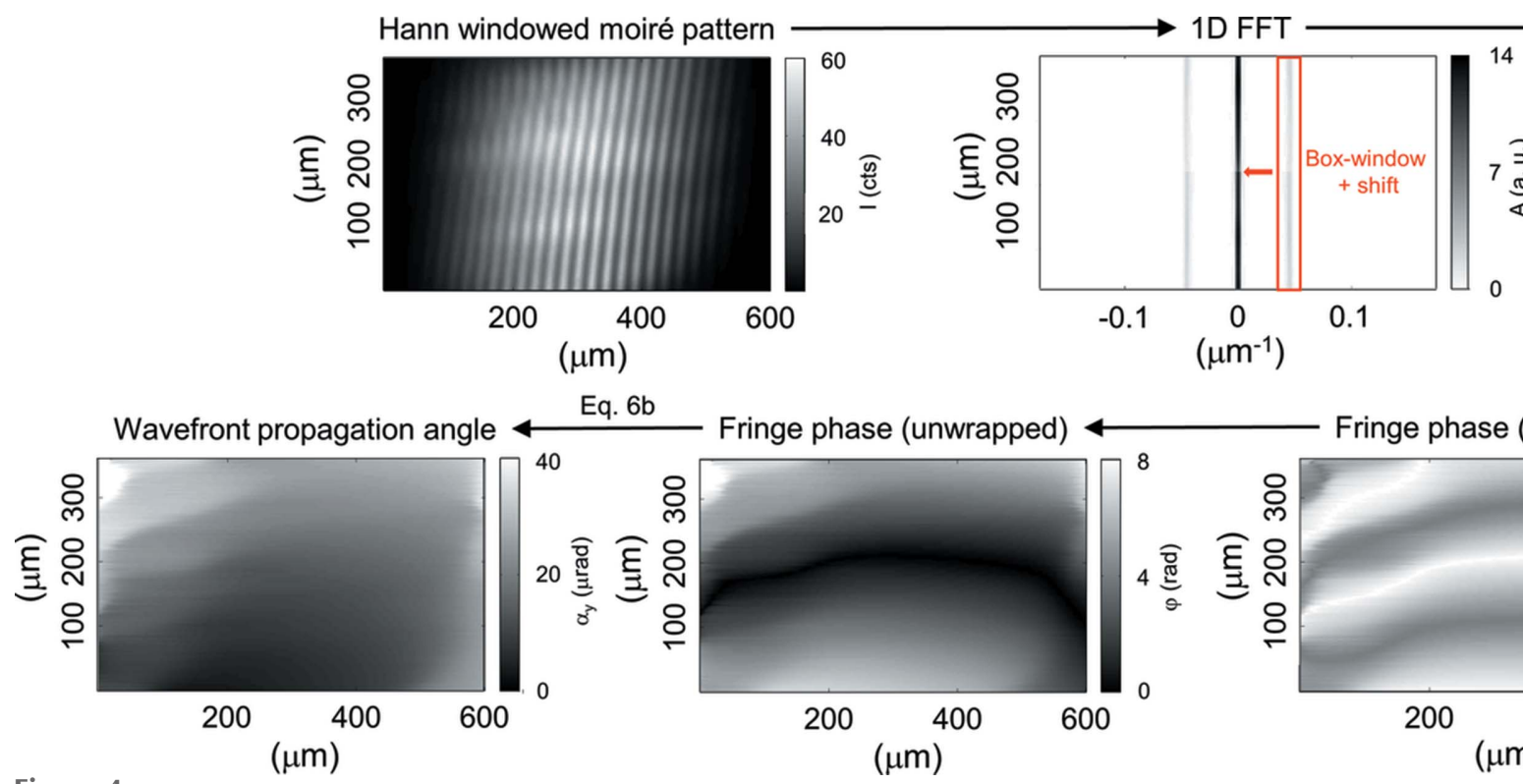

Figure 4

Flowchart of the Fourier analysis algorithm of a moiré pattern recorded with horizontally aligned line gratings. The wavefront propagation angle only contains the vertical component. In order to retrieve the horizontal wavefront propagation angle, either the line gratings have to be turned by $90^{\circ}$ or twodimensional gratings have to used. More details can be found in the text. 
If a reflective optical surface is mounted in front of the XGI setup, its height profile can be calculated through a projection of the aspherical component of the wavefront phase $\Phi$,

$$
h=\frac{\Phi \lambda}{4 \pi \tan \theta},
$$

where $\theta$ corresponds to the incidence angle of the $\mathrm{X}$-ray beam on the optical element. Propagation effects can be neglected for short distances between the optical element and the XGI setup. The wavefront phase $\Phi$ is obtained through an integration of the wavefront propagation angle. And a consecutive subtraction of a second-order polynomial yields its aspherical component.

The described calibration and analysis procedure for oneand two-dimensional gratings has been implemented into a Matlab environment which requires only the experimental parameters of the measurement as inpout. This reduces the user interaction to a minimum since all scripts and functions are called and executed hereafter automatically until the final results are returned. An even closer integration with the image acquisition and motor control interfaces is foreseen in future in order to develop the setup towards an efficient stand-alone tool with an online analysis toolbox. This will allow for a flexible and easy use of the XGI setup as a metrology tool for online wavefront diagnostic purposes independently from any beamline's hardware interface. A few exemplary applications will be presented hereafter.

\section{Metrology experiments}

Unless otherwise indicated, the measurements at the X05DA Optics beamline (Flechsig et al., 2009) were carried out at energies of $8.3 \mathrm{keV}$ or $12.4 \mathrm{keV}$ and in the fifth fractional Talbot order which is a typical choice for measurements at synchrotron radiation facilities. The gratings were usually horizontally oriented gratings since the monochromator and the mirror are both vertically deflecting. The XGI setup was installed at $\sim 16 \mathrm{~m}$ from the bending magnet of the beamline. Thus, the XGI setup was located about $8.9 \mathrm{~m}$ downstream from the monochromator and about $8.2 \mathrm{~m}$ from the bendable focusing mirror. The acquisition time per image was $15 \mathrm{~s}$ with non-focused and $0.1 \mathrm{~s}$ with focused X-rays. The analyzed wavefront and height profile sections are limited by upstream slits (1/6 mrad acceptance) and the incidence angle on the surface of the optical elements.

From the calibration measurements (Fig. 3), a vertical ROC of $15.9 \pm 0.3 \mathrm{~m}$ was obtained. This value matches well with the estimated distance to the bending magnet. Since the distance to the source point of the beamline and the ROC agree well, this indicates that the monochromator has as expected no focusing effect on the X-ray beam. Between successive measurements with a different angular stepping in the calibration scan but with an identical scanned angular range, the retrieved value for the ROC varied by only $0.2 \mathrm{~m}$. The angular position of the grating which was not moved during a calibration measurement was fitted with an error of $0.017^{\circ}$ and the maximum discrepancy in the fitted position between different scans was $0.014^{\circ}$. For vertically aligned gratings a horizontal $\mathrm{ROC}$ of $16.4 \pm 0.4 \mathrm{~m}$ was found. This agreement with the vertical ROC and with the distance to the X-ray source indicates again that the influence of the monochromator on the wavefront is minimal and that the curvature of the wavefront is essentially due to the propagation from the source point.

This conclusion was confirmed by the investigation of the moire patterns recorded at a single, fixed position of the gratings. The retrieved horizontal and vertical ROC were identical within the errors returned from the evaluation of calibration measurement. Furthermore, only small deviations from the spherical shape can be observed from the recorded moiré pattern which is essentially characterized by parallel undistorted fringes. In Fig. 5 the vertical component of the wavefront (horizontally aligned gratings) and the combined height profile of the channel-cut monochromator surfaces are shown for different angular orientations of the absorption grating (photon energy of $12.4 \mathrm{keV}$ ). Both the overall shape of the wavefront and that of the height profile do not change considerably. The aspherical component is fairly flat, indicating a well preserved wavefront, while the height profile yields a very smooth, flat surface without any pronounced height variations. The measured root-mean-square (RMS) wavefront aberration varies between $0.11 \mu \mathrm{rad}$ and $0.13 \mu \mathrm{rad}$ while the retrieved peak-to-valley $(\mathrm{PV})$ value is of the order of
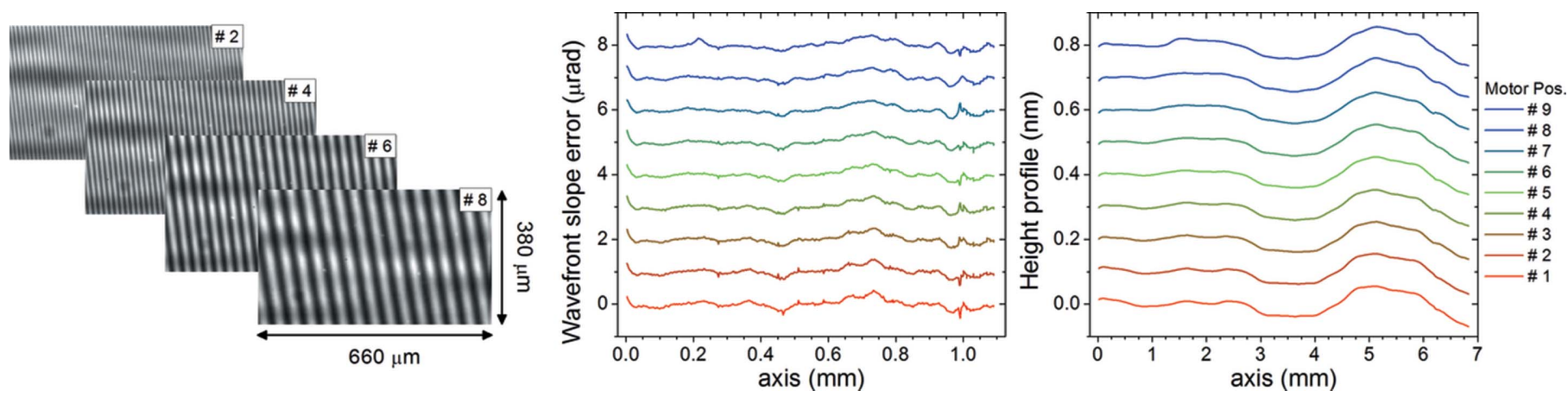

Figure 5

Vertical wavefront slope error and monochromator height profile reconstructed from moiré patterns recorded at different angular orientations around the optical axis of the absorption grating with respect to the diffraction grating. The data are plotted with vertical offsets of $1 \mu$ rad and $0.1 \mathrm{~nm}$, respectively. The results are independent of the grating position. Details of the recorded patterns are shown in the left-hand panel. 
$0.1 \mathrm{~nm}$. Note that the contributions from the two surfaces of the channel-cut monochromator cannot be separated. In order to verify the repeatability of a measurement the change in the RMS wavefront aberration and in the PV value of the height profile were monitored for 600 consecutive images at a fixed position of the gratings. The RMS of the wavefront aberration varied around $0.10 \mu \mathrm{rad}$ and the ROC around $16.0 \mathrm{~m}$. The standard variation of the indicated values are well below $0.01 \mu \mathrm{rad}$ and $0.1 \mathrm{~m}$. This indicates not only that the wavefront upstream of the interferometer is stable over time but also that the XGI technique allows for a reliable and accurate investigation of the wavefront. Given that the wavefront presents only small aberrations, the present configuration was used for more extensive investigations of the XGI setup.

Next, the influence of the gratings on the extracted X-ray wavefront and monochromator height profile was systematically studied at a photon energy of $8.3 \mathrm{keV}$ by rotating one of the gratings by $180^{\circ}$ either around the beam propagation axis or around an axis parallel to the grating structures. In the second case the upstream surface of the grating will become the downstream surface and vice versa. In total this makes up for 16 different arrangements. For each one a calibration measurement was realised and the moiré pattern was then investigated at a comparable angular orientation of the grating structures. The goal was to verify whether a correlation between the shape of the measured wavefront and the arrangement of the gratings can be observed. The results are shown in Fig. 6. Both the aspherical component of the wavefront and the height profile show a dependence on the grating orientation: the RMS aberration varies on a range from $0.11 \mu \mathrm{rad}$ to $0.20 \mu \mathrm{rad}$, and the height profile between 0 and $0.2 \mathrm{~nm}$. One reason for these pronounced changes could be traced back to a small defect of $120 \mu \mathrm{m} \times 35 \mu \mathrm{m}$ in the absorption grating which was present in the recorded moiré pattern in half of the measurements. For the measurements where this defect was not present in the field of view of the camera, the RMS aberration of the wavefront varied from $0.11 \mu \mathrm{rad}$ to $0.17 \mu \mathrm{rad}$ and the PV value of the height profile around $0.1 \mathrm{~nm}$. Thus, for the present measurement, the observed grating defect contributed to an increase in the RMS wavefront aberration of about $0.03 \mu \mathrm{rad}$. Consequently this defect was avoided in other measurements by arranging the gratings accordingly and displacing the XGI setup vertically if necessary. Without this defect, the measured wavefront was estimated to be independent of the grating arrangement and a possible impact of the gratings on the measurement was considered to be negligible. This underlines the importance of systematic control measurements of the grating quality. Another approach for checking the impact of the gratings on the experimental results is to displace the gratings laterally through the beam in the direction transverse to the grating structures and verify whether the recorded moiré pattern changes with the grating position. This possibility was, however, not implemented in the presented setup.

In a further set of measurements at $8.3 \mathrm{keV}$ the fractional Talbot order was changed by varying accordingly the intergrating distance (Fig. 7). This could be conveniently realised

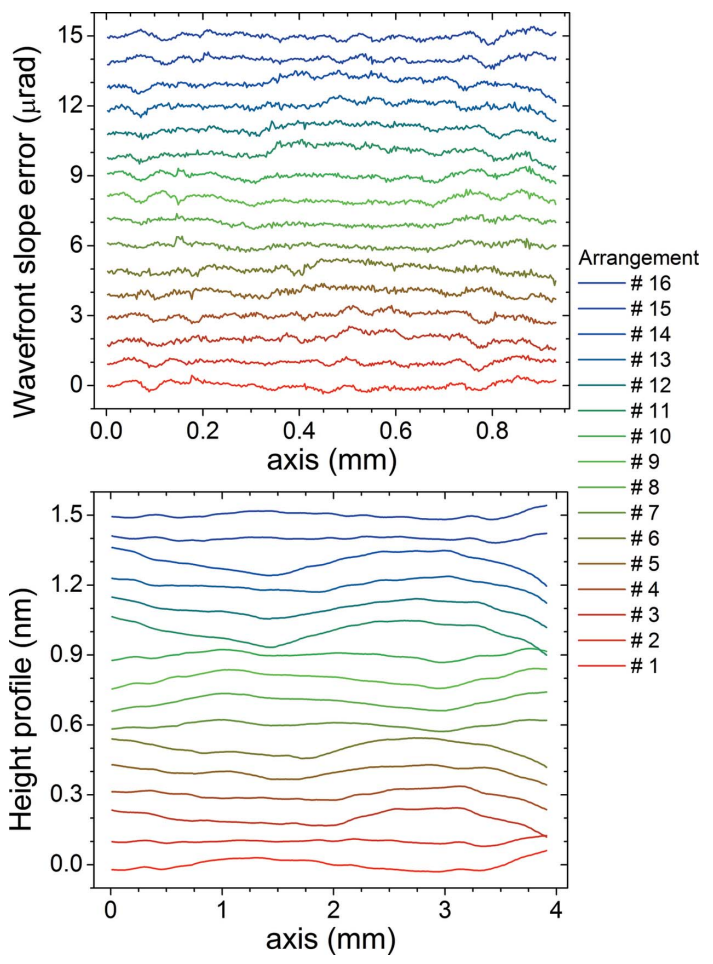

Figure 6

Wavefront slope error and monochromator height profile (plotted with vertical offsets of $1 \mu \mathrm{rad}$ and $0.1 \mathrm{~nm}$, respectively) retrieved from moiré patterns recorded with different arrangements of the gratings (details can be found in the text). The differences in the experimental results are attributed to a defect in the absorption grating.

with the presented setup, only the ninth could not be accessed because of spatial constraints imposed by the position of the holders. For each Talbot order a calibration measurement was realised and the moiré patterns analyzed at a comparable angular orientation of the gratings. The visibility of the moiré fringe pattern decreased with an increasing fractional Talbot order due to the limited transverse coherence of the X-ray beam leading to a blurring of the contrast in the interference pattern with increasing fractional Talbot order. Furthermore, the features visible in the height profile retrieved from the measurements at the fractional Talbot orders 5 and 7 wash out with increasing Talbot orders indicating that the spatial resolution decreases. For fractional Talbot orders larger than 33, the moiré patterns could not be analyzed due to lacking contrast in the fringe pattern. At fractional Talbot orders 31 and 33, artifacts in the aspherical wavefront and the height profile started to appear. Overall, the RMS value of the wavefront aberration was the largest for the third fractional Talbot order $(0.35 \mu \mathrm{rad})$ and presented afterwards a decreasing trend with the fractional Talbot order from around $0.17 \mu \mathrm{rad}$ to about $0.13 \mu \mathrm{rad}$ and was again larger for the $33 \mathrm{rd}$ fractional Talbot order. Also, for the PV value of the height profile a markedly larger value was found for the third fractional Talbot order $(0.4 \mathrm{~nm})$ followed by a slightly decreasing trend from $0.2 \mathrm{~nm}$ to $0.1 \mathrm{~nm}$. This can most likely be attributed to the decreasing spatial resolution (Weitkamp et al., 2005a,b).

For a complementary measurement, the image acquisition system was mounted together with the absorption grating on a 

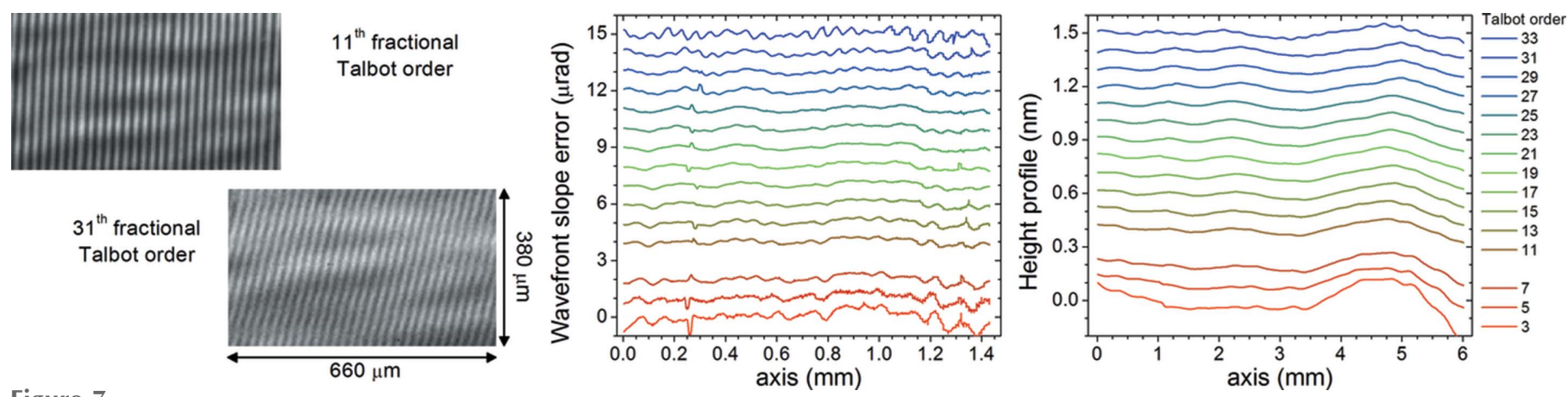

Figure 7

Wavefront slope error (vertical offset of $1 \mu \mathrm{rad}$ ) and monochromator height profile (vertical offset of $0.1 \mathrm{~nm}$ ) obtained from moiré patterns recorded at different fractional Talbot orders showing that the spatial resolution of the measurement decreases with increasing fractional Talbot order. Details of the recorded moiré patterns are shown in the left-hand panel for two selected fractional Talbot orders. The ninth fractional Talbot order was not accessible because of the arrangement of the holders.

linear stage (not shown in Fig. 2) such that the distance to the diffraction grating could be freely chosen along the beam propagation direction (Fig. 8). This measurement was realised with horizontally and with vertically aligned grating structures. The fringe visibility was defined as the ratio of the amplitude of the first Fourier order to the one of the zeroth order. The visibility oscillates with the intergrating distance and presents maximum values at the different fractional Talbot orders. As described by Diaz et al. (2010), from the envelope of the change in visibility the source size and the transverse coherence of the beam can be extracted. By following the same approach a transverse coherence length of $39.6 \mu \mathrm{m}$ (FWHM) and a source size of $25.0 \mu \mathrm{m}$ (FWHM) was found in the vertical direction. The corresponding values in the horizontal direction are $11.3 \mu \mathrm{m}$ and $86.9 \mu \mathrm{m}$. From the machine parameters the designed source size is calculated to be $27 \mu \mathrm{m}$ and $86 \mu \mathrm{m}$ (assumed coupling factor: 0.01 ). This shows that XGI is not only a suitable X-ray wavefront investigation tool but can also be used to characterize the coherence of the X-ray beam at a beamline, including the effect of all the elements in the beam transport system like monochromators, mirrors or
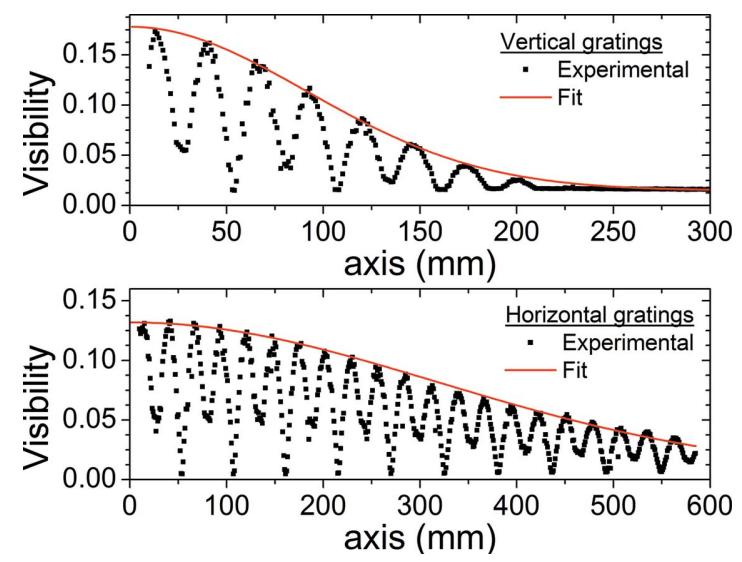

Figure 8

Measured change in visibility when varying the distance between the gratings for vertically and horizontally aligned line gratings (upper and lower panel, respectively). The different fractional Talbot orders can be recognized and the fit of the envelope allows the coherence of the X-ray beam to be characterized. windows. This kind of measurement, however, requires sufficient space along the beam path, e.g. at undulator beamlines travel ranges of the order of $1 \mathrm{~m}$ are needed (Diaz et al., 2010; Assoufid et al., 2016). The additionally required linear stage can be connected to the motion controller system so that the criterion of a self-contained and transportable setup is still satisfied.

The performance of the used gratings, which were designed for an energy of $8.3 \mathrm{keV}$, was also tested when modifying the photon energy while adjusting the intergrating distance to that of the fifth fractional Talbot order while assuming a $\pi$-phase shifting grating (Fig. 9). Although, the phase shift induced by the diffraction grating and the position of the different fractional Talbot orders depend on the X-ray photon energy, the fringe pattern itself is less sensitive to a change in the photon energy. For a fixed distance between the diffraction and absorption grating a deviation of the X-ray photon energy from the design energy of the XGI setup only leads to a decreased fringe visibility. It turned out that the gratings could be used over an energy range of almost $4 \mathrm{keV}$; the recorded moiré pattern could still be reliably analyzed. The limitations were the diminishing visibility of the moiré fringes when increasing the energy difference to the design energy and the inherently increasing influence of noise in the recorded moiré patterns. The change in visibility with photon energy could be well reproduced with a Gaussian function (maximum at $8.4 \mathrm{keV}$, width of $1.9 \mathrm{keV}$ ). This was mainly due to a deviation of the phase-shift induced in the grating structures from the optimum value of $\pi$ and to a lesser level to the fixed intergrating distance. The efficiency of the diffraction gratings at photon energies larger than the design energy could be improved by rotating the gratings around an axis parallel to the orientation of the grating structures and adjusting the intergrating distance since the position of the fractional Talbot orders depends on the photon energy. The option to rotate the gratings around a different axis than the beam propagation axis was, however, not foreseen in the presented XGI setup but would allow covering an even larger range of photon energies with a single set of gratings.

Two more application-oriented measurements are presented in Figs. 10 and 11. In a first measurement the RMS 


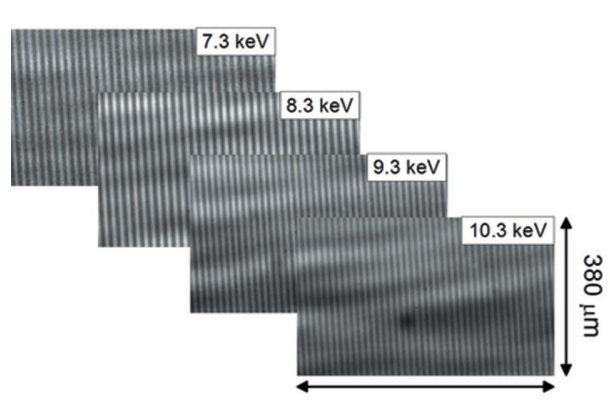

Figure 9
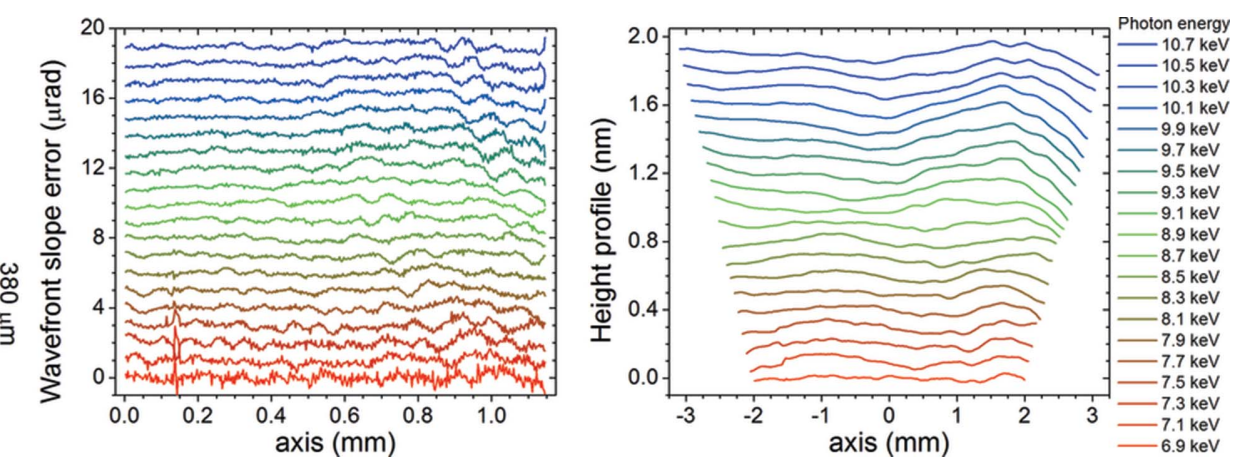

Wavefront slope error (vertical offset of $1 \mu \mathrm{rad}$ ) and monochromator height profile (vertical offset of $0.1 \mathrm{~nm}$ ) reconstructed from moiré patterns recorded at different incident photon energies. Details of the recorded moiré patterns are shown in the left-hand panel. The intergrating distance was kept fixed and the design energy of the gratings was $8.3 \mathrm{keV}$.
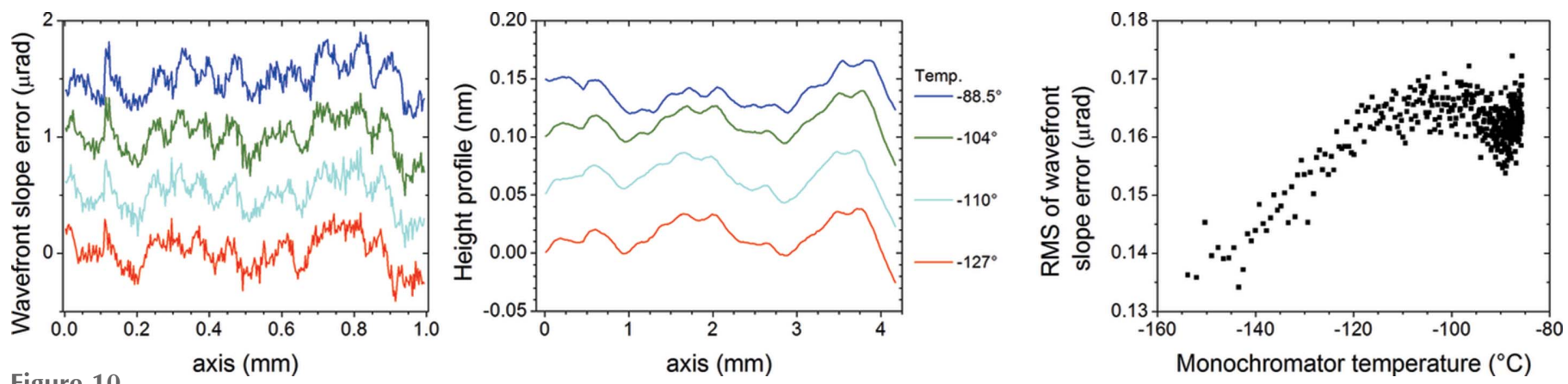

Wavefront slope error and monochromator height profile retrieved from moiré patterns recorded at different temperatures of the cryogenically cooled monochromator and plotted with vertical offsets (left-hand and middle panel). As the temperature rises a slight increase in the RMS value of the aspherical wavefront component was observed (right-hand panel).

aberration of the aspherical wavefront component is monitored as the temperature of the cryogenically cooled monochromator increases (Fig. 10). Before the measurement the front-end slits were kept closed for several hours in order to have a stable temperature of the monochromator. The change in the wavefront aberration was then followed over time by measuring successively the moiré patterns until a stable temperature condition was reached. Thus, the XGI setup can be used to follow changes in the quality of the wavefront induced by the power of the incident radiation on the optical components. For these type of measurements, the moiré mode is highly suited since the time resolution is only limited by the acquisition time for a single image. In a second application the quality of the wavefront from a small 1-inch $\mathrm{Si}$ substrate coated with $10 \mathrm{~nm}$ Ir on top of $5 \mathrm{~nm} \mathrm{Mo}$ and $15 \mathrm{~nm} \mathrm{~B}{ }_{4} C$ was measured (Fig. 11). The sample was mounted on the head of the goniometer attached to the diffractometer on which the XGI setup was installed. The sample could be inserted and retracted from the beam so that a comparison with the wavefront with and without the sample in the beam could be realised. The incidence angle of the X-ray beam was $0.16^{\circ}$ which limited the spatial resolution of the retrieved height profile. It was observed that the aspherical component of the wavefront from the beamline was negligible so that an investigation of the sample was possible without correcting the beamline contribution with a flat-field measurement. The height profile reveals a PV value of $2.2 \mathrm{~nm}$. This shows that the
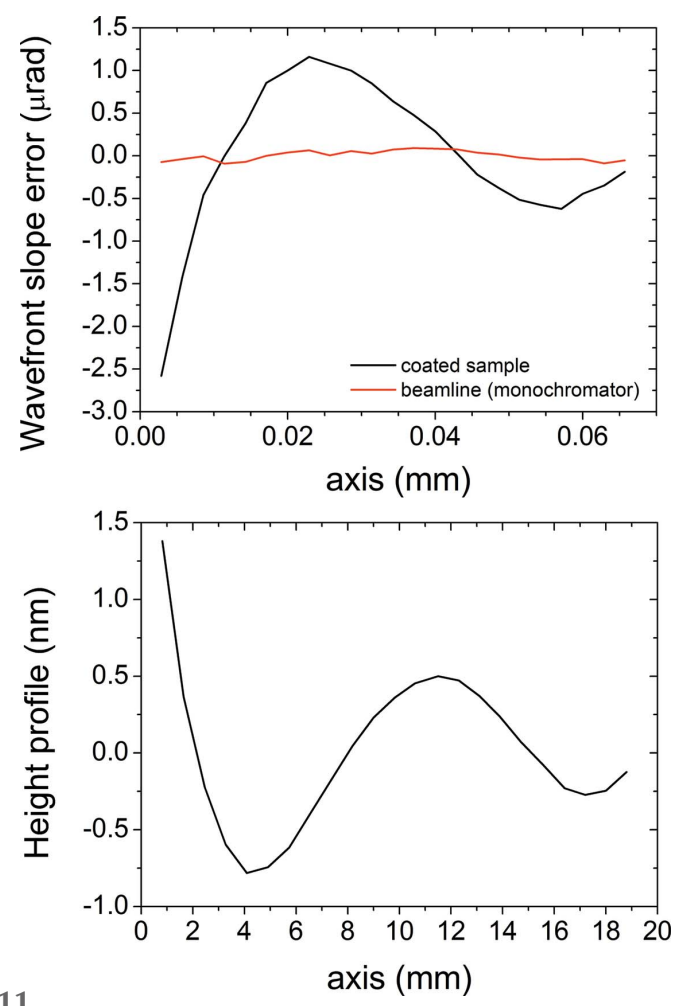

Figure 11

Wavefront slope error (top panel) and height profile (bottom panel) of a $\mathrm{Si}$ wafer coated with $10 \mathrm{~nm}$ Ir on top of $5 \mathrm{~nm}$ Mo and $15 \mathrm{~nm} \mathrm{~B}_{4} \mathrm{C}$. The wavefront slope error from the monochromated synchrotron beam incident on the multilayered sample is shown for comparison (top panel). 

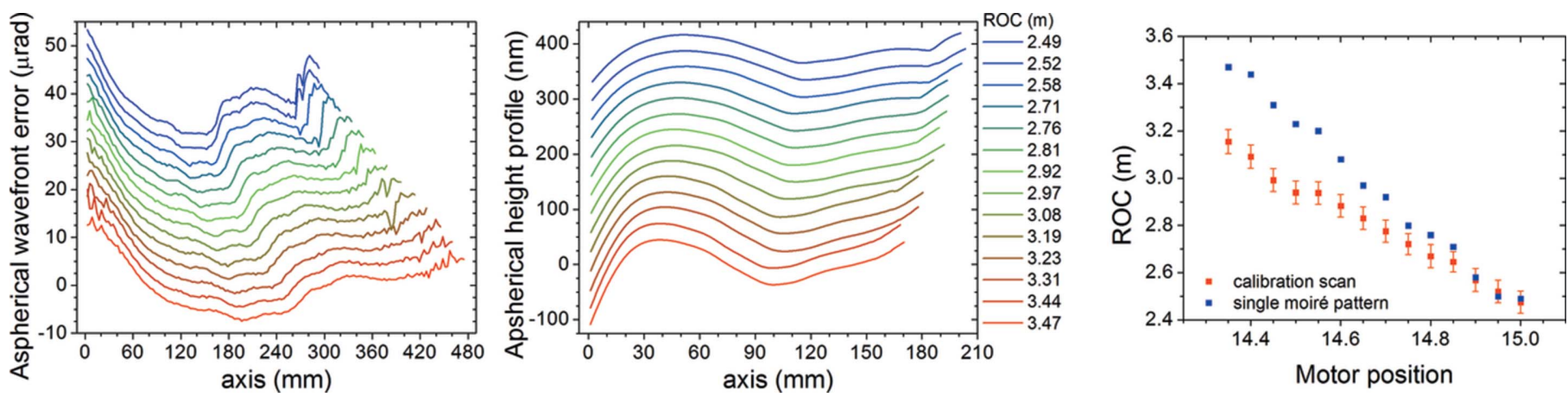

Figure 12

Wavefront slope error and monochromator height profile retrieved for different bending positions of the focusing mirror (left-hand and middle panel). Data sets are vertically offset for clarity. The ROC can be calculated for each position and indicates the focal position with respect to the XGI setup (right-hand panel).

XGI setup has the potential for at-wavelength investigations of reflection mirrors.

The bendable focusing mirror of the X05DA Optics beamline could be analyzed as well at an energy of $8.3 \mathrm{keV}$ with the installed XGI setup. Since the XGI setup was used in the moire mode, the same set of gratings as for the unfocused $\mathrm{X}$-ray beam could be used despite the divergence of the beam downstream of the focal position. The working distance of the mirror is about $8 \mathrm{~m}$. The bendable mirror allows the focal position to be moved or the X-ray fluence to be tuned at a given position along the beam propagation axis. The wavefront and the height profile of the mirror were extracted for different bending positions of the mirror where the focal position of the mirror was upstream of the XGI setup (Fig. 12). Since the monochromator was found to not introduce significant aberrations into the aspherical component of the wavefront, the observed aberrations can be attributed to the focusing mirror. The RMS of the wavefront was found to vary around $5.40 \mu \mathrm{rad}$ for the investigated positions. From the moiré pattern the ROC of the X-ray wavefront can be extracted as well. This allowed us to determine the focus position of the bendable mirror with respect to the XGI setup as shown in Fig. 12. The focus position was scanned for different positions upstream of the XGI setup. At each position a calibration measurement was realised and the moiré patterns recorded for 50 images at a single position of the gratings. This allows the focal position with respect to the XGI setup to be retrieved for different positions of the bender and thus the corresponding motor to be calibrated. For focal positions too close to the XGI setup the moiré patterns could not be analyzed because of lacking contrast of the fringes (visibility below $2.5 \%$ ) and the small spot size. Thus, the presented type of measurements demonstrates that the described XGI setup is well suited for the characterization and tuning of adaptive optics.

For reasons of completeness it should be mentioned that, for focusing optics with short working distances producing $\mathrm{X}$-ray beams with a pronounced divergence, single-grating setups present an option since the divergence produces a sufficient magnification to resolve the interference fringes with an adequate camera system (Yumoto et al., 2006; Yuan et al.,
2011; Nilsson et al., 2012; Matsuyama et al., 2012; Berujon \& Ziegler, 2012; Fukui et al., 2013; Uhlén et al., 2014). Otherwise, the diffraction and the absorption grating need to be divergence-matched in order to account for the magnification of the interference fringes induced by the beam divergence.

\section{Conclusion}

In summary, the reported XGI setup presents a valuable atwavelength diagnostic tool for quantitative, spatially resolved $\mathrm{X}$-ray wavefront metrology measurements at synchrotron radiation beamlines or XFEL endstations. In the presented measurements we demonstrated that the high angular sensitivity of XGI can be used for routine in situ investigations of the X-ray wavefront. The described XGI setup is flexible in use, can be easily taken into operation and only presents moderate space requirements for the installation. The convenient access to in situ and at-wavelength wavefront metrology tools like XGI is indeed highly desirable in order to enable the determination of wavefront distortions caused for example by the mechanical mounting or the incident X-ray power. In this view, the presented XGI setup can be considered as a valuable diagnostic instrument for the evaluation and improvement of the performance of optical elements and ultimately support the development of X-ray optics able to preserve the wavefront shape and the coherence of the X-ray beam delivered by the X-ray source. Possible future applications of the XGI setup are the adjustment of adaptive optics like bendable mirrors, for example, the validation of beam transport simulation, or cross-comparison campaigns with other in situ and ex situ metrology techniques.

\section{Acknowledgements}

The authors acknowledge the Swiss Light Source at the Paul Scherrer Institut for providing beam time. The experiments were performed at the X05DA Optics beamline. We acknowledge the technical support of Andreas Jaggi and Vincent Thominet and thank Rolf Follath for providing the mulitlayer sample. 


\section{References}

Alcock, S., Sawhney, K., Scott, S., Pedersen, U., Walton, R., Siewert, F., Zeschke, T., Senf, F., Noll, T. \& Lammert, H. (2010). Nucl. Instrum. Methods. Phys. Res. A, 616, 224-228.

Assoufid, L., Shi, X., Marathe, S., Benda, E., Wojcik, M. J., Lang, K., Xu, R., Liu, W., Macrander, A. T. \& Tischler, J. Z. (2016). Rev. Sci. Instrum. 87, 052004.

Berujon, S. \& Ziegler, E. (2012). Opt. Lett. 37, 4464-4466.

Berujon, S., Ziegler, E., Cerbino, R. \& Peverini, L. (2012). Phys. Rev. Lett. 108, 158102.

David, C., Bruder, J., Rohbeck, T., Grünzweig, C., Kottler, C., Diaz, A., Bunk, O. \& Pfeiffer, F. (2007). Microelectron. Eng. 84, 11721177.

David, C. \& Hambach, D. (1999). Microelectron. Eng. 46, 219-222.

David, C., Nöhammer, B., Solak, H. H. \& Ziegler, E. (2002). Appl. Phys. Lett. 81, 3287-3289.

Diaz, A., Mocuta, C., Stangl, J., Keplinger, M., Weitkamp, T., Pfeiffer, F., David, C., Metzger, T. H. \& Bauer, G. (2010). J. Synchrotron Rad. 17, 299-307.

Emma, P., Akre, R., Arthur, J., Bionta, R., Bostedt, C., Bozek, J., Brachmann, A., Bucksbaum, P., Coffee, R., Decker, F.-J., Ding, Y., Dowell, D., Edstrom, S., Fisher, A., Frisch, J., Gilevich, S., Hastings, J., Hays, G., Hering, P., Huang, Z., Iverson, R., Loos, H., Messerschmidt, M., Miahnahri, A., Moeller, S., Nuhn, H.-D., Pile, G., Ratner, D., Rzepiela, J., Schultz, D., Smith, T., Stefan, P., Tompkins, H., Turner, J., Welch, J., White, W., Wu, J., Yocky, G. \& Galayda, J. (2010). Nat. Photon. 4, 641-647.

Eriksson, M., van der Veen, J. F. \& Quitmann, C. (2014). J. Synchrotron Rad. 21, 837-842.

Flechsig, U., Jaggi, A., Krempaský, J., Spielmann, S. \& Thominet, V. (2013). Nucl. Instrum. Methods Phys. Res. A, 710, 13-16.

Flechsig, U., Jaggi, A., Spielmann, S., Padmore, H. \& MacDowell, A. (2009). Nucl. Instrum. Methods. A, 609, 281-285.

Fukui, R., Kim, J., Matsuyama, S., Yumoto, H., Inubushi, Y., Tono, K., Koyama, T., Kimura, T., Mimura, H., Ohashi, H., Yabashi, M., Ishikawa, T. \& Yamauchi, K. (2013). Synchrotron Radiat. News, 26, 13-16.

Goldberg, K. A., Yashchuk, V. V., Artemiev, N. A., Celestre, R., Chao, W., Gullikson, E. M., Lacey, I., McKinney, W. R., Merthe, D. \& Padmore, H. A. (2013). Synchrotron Radiat. News, 26, 4-12.

Hariharan, P. (1997). Opt. Eng. 36, 2478-2481.

Herráez, M. A., Burton, D. R., Lalor, M. J. \& Gdeisat, M. A. (2002). Appl. Opt. 41, 7437-7444.

Hignette, O., Freund, A. K. \& Chinchio, E. (1997). Proc. SPIE, 3152, 188-199.

Idir, M., Dovillaire, G. \& Mercere, P. (2013). Synchrotron Radiat. News, 26, 23-29.

Idir, M., Kaznatcheev, K., Dovillaire, G., Legrand, J. \& Rungsawang, R. (2014). Opt. Express, 22, 2770-2781.

Ishikawa, T., Aoyagi, H., Asaka, T., Asano, Y., Azumi, N., Bizen, T., Ego, H., Fukami, K., Fukui, T., Furukawa, Y., Goto, S., Hanaki, H., Hara, T., Hasegawa, T., Hatsui, T., Higashiya, A., Hirono, T., Hosoda, N., Ishii, M., Inagaki, T., Inubushi, Y., Itoga, T., Joti, Y., Kago, M., Kameshima, T., Kimura, H., Kirihara, Y., Kiyomichi, A., Kobayashi, T., Kondo, C., Kudo, T., Maesaka, H., Maréchal, X. M., Masuda, T., Matsubara, S., Matsumoto, T., Matsushita, T., Matsui, S., Nagasono, M., Nariyama, N., Ohashi, H., Ohata, T., Ohshima, T., Ono, S., Otake, Y., Saji, C., Sakurai, T., Sato, T., Sawada, K., Seike, T., Shirasawa, K., Sugimoto, T., Suzuki, S., Takahashi, S., Takebe, H., Takeshita, K., Tamasaku, K., Tanaka, H., Tanaka, R., Tanaka, T., Togashi, T., Togawa, K., Tokuhisa, A., Tomizawa, H., Tono, K., Wu, S., Yabashi, M., Yamaga, M., Yamashita, A., Yanagida, K., Zhang, C., Shintake, T., Kitamura, H. \& Kumagai, N. (2012). Nat. Photon. 6, 540-544.

Kayser, Y., Rutishauser, S., Katayama, T., Kameshima, T., Ohashi, H., Flechsig, U., Yabashi, M. \& David, C. (2016). Opt. Lett. 41, 733-736.
Kayser, Y., Rutishauser, S., Katayama, T., Ohashi, H., Kameshima, T., Flechsig, U., Yabashi, M. \& David, C. (2014). Opt. Express, 22, 9004-9015.

Marathe, S., Shi, X., Khounsary, A. M., Wojcik, M. J., Kujala, N. G., Macrander, A. T. \& Assoufid, L. (2014a). Proc. SPIE, 9208, 92080D.

Marathe, S., Shi, X., Wojcik, M. J., Kujala, N. G., Divan, R., Mancini, D. C., Macrander, A. T. \& Assoufid, L. (2014b). Opt. Express, 22, 14041-14053.

Matsuyama, S., Yokoyama, H., Fukui, R., Kohmura, Y., Tamasaku, K., Yabashi, M., Yashiro, W., Momose, A., Ishikawa, T. \& Yamauchi, K. (2012). Opt. Express, 20, 24977-24986.

Momose, A., Kawamoto, S., Koyama, I., Hamaishi, Y., Takai, K. \& Suzuki, Y. (2003). Jpn. J. Appl. Phys. 42, L866.

Momose, A., Yashiro, W., Takeda, Y., Suzuki, Y. \& Hattori, T. (2006). Jpn. J. Appl. Phys. 45, 5254.

Nilsson, D., Uhlén, F., Holmberg, A., Hertz, H. M., Schropp, A., Patommel, J., Hoppe, R., Seiboth, F., Meier, V., Schroer, C. G., Galtier, E., Nagler, B., Lee, H. J. \& Vogt, U. (2012). Opt. Lett. 37, 5046-5048.

Noda, D., Tanaka, M., Shimada, K., Yashiro, W., Momose, A. \& Hattori, T. (2008). Microsyst Technol. 14, 1311-1315.

Pfeiffer, F., Bunk, O., Schulze-Briese, C., Diaz, A., Weitkamp, T., David, C., van der Veen, J. F., Vartanyants, I. \& Robinson, I. K. (2005). Phys. Rev. Lett. 94, 164801.

Rack, A., Weitkamp, T., Riotte, M., Grigoriev, D., Rack, T., Helfen, L., Baumbach, T., Dietsch, R., Holz, T., Krämer, M., Siewert, F., Meduna, M., Cloetens, P. \& Ziegler, E. (2010). J. Synchrotron Rad. 17, 496-510.

Reznikova, E., Mohr, J., Boerner, M., Nazmov, V. \& Jakobs, P.-J. (2008). Microsyst Technol. 14, 1683-1688.

Rivers, M. L., Garrett, R., Gentle, I., Nugent, K. \& Wilkins, S. (2010). AIP Conf. Proc. 1234, 51-54.

Romano, L., Kagias, M., Jefimovs, K. \& Stampanoni, M. (2016). RSC Adv. 6, 16025-16029.

Rutishauser, S., Bednarzik, M., Zanette, I., Weitkamp, T., Börner, M., Mohr, J. \& David, C. (2013b). Microelectron. Eng. 101, 12-16.

Rutishauser, S., Rack, A., Weitkamp, T., Kayser, Y., David, C. \& Macrander, A. T. (2013a). J. Synchrotron Rad. 20, 300-305.

Rutishauser, S., Samoylova, L., Krzywinski, J., Bunk, O., Grünert, J., Sinn, H., Cammarata, M., Fritz, D. M. \& David, C. (2012). Nat. Commun. 3, 947.

Rutishauser, S., Zanette, I., Donath, T., Sahlholm, A., Linnros, J. \& David, C. (2011). Appl. Phys. Lett. 98, 171107.

Sawhney, K., Wang, H., Sutter, J., Alcock, S. \& Berujon, S. (2013). Synchrotron Radiat. News, 26, 17-22.

Schnabel, B. \& Kley, E.-B. (2001). Microelectron. Eng. 57-58, $327-$ 333.

Shi, X., Marathe, S., Wojcik, M. J., Kujala, N. G., Macrander, A. T. \& Assoufid, L. (2014). Appl. Phys. Lett. 105, 041116.

Siewert, F., Buchheim, J., Zeschke, T., Brenner, G., Kapitzki, S. \& Tiedtke, K. (2011). Nucl. Instrum. Methods Phys. Res. A, 635, S52-S57.

Siewert, F., Buchheim, J., Zeschke, T., Störmer, M., Falkenberg, G. \& Sankari, R. (2014). J. Synchrotron Rad. 21, 968-975.

Störmer, M., Siewert, F. \& Sinn, H. (2016). J. Synchrotron Rad. 23, $50-58$.

Suleski, T. J. (1997). Appl. Opt. 36, 4686-4691.

Sutter, J., Alcock, S. \& Sawhney, K. (2012). J. Synchrotron Rad. 19, 960-968.

Takeda, M., Ina, H. \& Kobayashi, S. (1982). J. Opt. Soc. Am. 72, 156160.

Thiess, H., Lasser, H. \& Siewert, F. (2010). Nucl. Instrum. Methods. A, 616, 157-161.

Uhlén, F., Rahomäki, J., Nilsson, D., Seiboth, F., Sanz, C., Wagner, U., Rau, C., Schroer, C. G. \& Vogt, U. (2014). J. Synchrotron Rad. 21, 1105-1109. 
Wang, H., Berujon, S., Pape, I., Rutishauser, S., David, C. \& Sawhney, K. (2013a). Nucl. Instrum. Methods Phys. Res. A, 710, 78-81.

Wang, H., Berujon, S., Pape, I., Rutishauser, S., David, C. \& Sawhney, K. (2013b). Opt. Lett. 38, 827-829.

Wang, H., Kashyap, Y., Laundy, D. \& Sawhney, K. (2015). J. Synchrotron Rad. 22, 925-929.

Wang, H., Sawhney, K., Berujon, S., Sutter, J., Alcock, S. G., Wagner, U. \& Rau, C. (2014). Opt. Lett. 39, 2518-2521.

Wang, H., Sawhney, K., Berujon, S., Ziegler, E., Rutishauser, S. \& David, C. (2011). Opt. Express, 19, 16550-16559.

Weitkamp, T., Diaz, A., David, C., Pfeiffer, F., Stampanoni, M., Cloetens, P. \& Ziegler, E. (2005b). Opt. Express, 13, 6296-6304.

Weitkamp, T., Diaz, A., Nöhammer, B., Pfeiffer, F., Stampanoni, M., Ziegler, E. \& David, C. (2004). Proc. SPIE, 5533, 140-144.

Weitkamp, T., Nöhammer, B., Diaz, A., David, C. \& Ziegler, E. (2005a). Appl. Phys. Lett. 86, 054101.

Weitkamp, T., Zanette, I., Pfeiffer, F. \& David, C. (2012). AIP Conf. Proc. 1466, 84-89.
Yabashi, M., Tono, K., Mimura, H., Matsuyama, S., Yamauchi, K., Tanaka, T., Tanaka, H., Tamasaku, K., Ohashi, H., Goto, S. \& Ishikawa, T. (2014). J. Synchrotron Rad. 21, 976-985.

Yamada, J., Matsuyama, S., Sano, Y. \& Yamauchi, K. (2015). Rev. Sci. Instrum. 86, 093103.

Yashchuk, V. V., Artemiev, N. A., Lacey, I., McKinney, W. R. \& Padmore, H. A. (2015). Opt. Eng. 54, 104104.

Yashchuk, V., Barber, S., Domning, E., Kirschman, J., Morrison, G., Smith, B., Siewert, F., Zeschke, T., Geckeler, R. \& Just, A. (2010). Nucl. Instrum. Methods. A, 616, 212-223.

Yuan, S., Goldberg, K. A., Yashchuk, V. V., Celestre, R., McKinney, W. R., Morrison, G., Macdougall, J., Mochi, I. \& Warwick, T. (2011). Nucl. Instrum. Methods Phys. Res. A, 635, S58-S63.

Yumoto, H., Mimura, H., Matsuyama, S., Handa, S., Sano, Y., Yabashi, M., Nishino, Y., Tamasaku, K., Ishikawa, T. \& Yamauchi, K. (2006). Rev. Sci. Instrum. 77, 063712.

Ziegler, E., Peverini, L., Kozhevnikov, I. V., Weitkamp, T. \& David, C. (2007). AIP Conf. Proc. 879, 778-781. 